\title{
Estimation of Organ Motion from 4D CT for 4D Radiation Therapy Planning of Lung Cancer
}

\author{
Michael R. Kaus ${ }^{1}$, Thomas Netsch ${ }^{1}$, Sven Kabus ${ }^{1,2}$, Vladimir Pekar ${ }^{1}$, Todd McNutt ${ }^{3}$, \\ and Bernd Fischer ${ }^{2}$ \\ 1 Philips Research Laboratories, Röntgenstr. 24-26, 22335 Hamburg, Germany \\ michael.kaus@philips . com \\ 2 Institute of Mathematics, University of Lübeck, Wallstraße 40, 23560 Lübeck, Germany \\ 3 Philips Medical Systems, 6400 Enterprise Lane, Madison, WI 53719, USA
}

\begin{abstract}
The goal of this paper is to automatically estimate the motion of the tumor and the internal organs from 4D CT and to extract the organ surfaces. Motion induced by breathing and heart beating is an important uncertainty in conformal external beam radiotherapy (RT) of lung tumors. 4D RT aims at compensating the geometry changes during irradiation by incorporating the motion into the treatment plan using 4D CT imagery. We establish two different methods to propagate organ models through the image time series, one based on deformable surface meshes, and the other based on volumetric B-spline registration. The methods are quantitatively evaluated on 8 3D CT images of the full breathing cycle of a patient with manually segmented lungs and heart. Both methods achieve good overall results, with mean errors of $1.02-1.33 \mathrm{~mm}$ and $0.78-2.05 \mathrm{~mm}$ for deformable surfaces and B-splines respectively. The deformable mesh is fast (40 seconds vs. 50 minutes), but accommodation of the heart and the tumor is currently not possible. B-spline registration estimates the motion of all structures in the image and their interior, but is susceptible to motion artifacts in CT.
\end{abstract}

Keywords: Radiation therapy, lung cancer, 4D CT, deformable registration, deformable surface models, B-splines

\section{Introduction}

External beam radiation therapy (RT) is one of the main cancer therapies for lung cancer, the leading cause of all cancer-related deaths with more than 150000 deaths in the USA each year [1]. Breathing and heart motion during irradiation causes significant variations in organ and target geometry in the order of several centimeters. This increases the dose to healthy tissue and reduces the dose to the target area, impairing the balance between complications and cure. 4D RT aims at compensating the deformation uncertainty by incorporating the motion characteristics into the dose calculation or gating the treatment device in phase with the motion pattern [2]. These techniques require a patient-specific motion model. With the advent of multi-slice CT, 4D image acquisition of dynamic processes such as breathing is now becoming possible.

Fully automated algorithms are desirable to estimate organ motion, but need to be sufficiently fast and with minimal user interaction [3]. A large variety of automated 
methods exists, including volumetric intensity-based registration techniques [4] and surface-based techniques [5]. Registration based on B-splines [6] has shown potential for medical applications such as breast MRI [7], brain [8] and cardiac [9]. Deformable surface models are computationally efficient, and their versatility has been demonstrated on a number of clinical applications including cardiac MRI time series [10[11] or brain mapping [12].

The goal of this paper is to establish and compare a volumetric and a surface based method for the estimation of internal organ motion as a first step towards 4D radiotherapy. The first method is a fast volumetric intensity-based B-spline registration method which has previously been applied to PET-CT registration and has not yet been quantitatively validated on clinical data [13]. The second approach is a deformable surface model algorithm which has previously been applied to bone segmentation in CT and left ventricle segmentation in MRI [14[11]. In the remainder of the text we will outline the two algorithms and their adaptation to 4D lung CT over the breathing cycle of one patient, and compare their results to manual segmentation of the lungs and the heart.

\section{Methods}

We apply two methods to automatically estimate organ motion in 4D images. The principle is to propagate $3 \mathrm{D}$ triangular surface meshes derived from a template image to the remaining images by re-calculation of the mesh vertex coordinates while leaving the mesh topology unchanged. The first approach is the volumetric grey-value based B-spline registration method described in [13], where the vertex coordinates are recomputed according to the $3 \mathrm{D}$ deformation field estimated for each voxel. The second method is the deformable surface mesh already proposed in [11], where the new vertex coordinates are calculated by minimization of the sum of an internal shape energy and an external feature energy.

\subsection{Volumetric Grey-Value Based B-Spline Registration}

For the transformation of a voxel position $\mathbf{x}_{v}=\left(x_{1}, x_{2}, x_{3}\right)^{\top}$ a grid $G$ of $g_{1} \times g_{2} \times g_{3}$ control points with uniform spacing in each direction is defined. The displacement of a single control point is encoded by $\beta_{i j k}$ and $\beta$ is the collection of all these vectors. Following Rueckert et al. [6] the B-spline deformation $\mathbf{u}$ at position $\mathbf{x}_{v}$ can be described by a tensor product

$$
\mathbf{u}\left(\mathbf{x}_{v} ; \beta\right)=\sum_{i=1}^{g_{1}} \sum_{j=1}^{g_{2}} \sum_{k=1}^{g_{3}} \beta_{i j k} b_{i, 3}\left(x_{1}\right) b_{j, 3}\left(x_{2}\right) b_{k, 3}\left(x_{3}\right)
$$

where $b_{,, 3}$ refers to a cubic B-spline. The transformation itself is defined as the subtraction of a deformation $\mathbf{u}\left(\mathbf{x}_{v} ; \beta\right)$ from its original position $\mathbf{x}_{v}$. The grid-and an extra layer of control points to avoid the use of boundary conditions-is placed on the image in such a way that the outmost grid controls coincide with the corners of the image.

For a given displacement field $\beta$, the quality of the match between the reference image $R$ and the image to be registered $T$ is determined by the sum $f$ of squared differences (SSD) over the total number of voxels $N$ : 


$$
f(\beta)=\frac{1}{2} \sum_{v=1}^{N}\left[F_{v}(\beta)\right]^{2}=\frac{1}{2}\|\mathbf{F}(\beta)\|_{2}^{2},
$$

where $F_{v}(\beta):=T\left(\mathbf{x}_{v}-\mathbf{u}\left(\mathbf{x}_{v}\right)\right)-R\left(\mathbf{x}_{v}\right)$ and $\mathbf{F}(\beta)=\left(F_{1}(\beta), \ldots, F_{N}(\beta)\right)^{\top}$. The optimization of the cost function $f$ needs special attention since the large number of parameters involved may result in high computational cost. We therefore choose an iterative Levenberg-Marquardt method, one of the most efficient non-linear optimization schemes, generating a sequence $\left(\beta^{m}\right)$ with starting value $\beta^{0} \equiv \mathbf{0}$ and update rule $\beta^{m+1}:=\beta^{m}+\alpha_{m} \mathbf{s}^{m}$. The parameter $\alpha_{m} \in[0,1]$ denotes the step-size and $\mathbf{s}^{m}$ the search direction respectively. The search direction $s^{m}$ is computed by solving the linear system

$$
\left[J_{F}\left(\beta^{m}\right)^{\top} J_{F}\left(\beta^{m}\right)+\lambda_{m} I\right] \mathbf{s}^{m}=-J_{F}\left(\beta^{m}\right)^{\top} \mathbf{F}\left(\beta^{m}\right),
$$

where $J_{F}\left(\beta^{m}\right)$ denotes the Jacobian of $\mathbf{F}\left(\beta^{m}\right)$ with entries $\partial F_{v}\left(\beta^{m}\right) / \partial \beta_{i}^{m}$. The diagonal matrix $\lambda_{m} I$ must be added to obtain a regular linear system since $J_{F}\left(\beta^{m}\right)$ is usually rank deficient: voxels in areas with constant or almost constant grey values yield zero partial derivatives [15]. The so-called trust region radius $\lambda_{m}$ can be considered as an adaption of the step-length during the iteration.

In each iteration step the matrix and the right hand side of (3) is built. The partial derivatives $\partial F_{v}\left(\beta^{m}\right) / \partial \beta_{i}^{m}$ are approximated by finite differences. In practice, many of the partial derivatives have a small numerical value and can be set to zero without degrading the convergence rate, but leading to a much more favorable complexity. For a given $\lambda_{m}$ the linear system is solved by a conjugate gradient scheme yielding the decent direction $\mathbf{s}^{m}$. Then the algorithm checks if the parameter update $\beta^{m+1}$ for $\alpha_{m}=1$ leads to a reduction in the cost function. If this is not the case $\alpha_{m}$ will be reduced stepwise until $f\left(\beta^{m}+\alpha_{m} \mathbf{s}^{m}\right)<0.995 f\left(\beta^{m}\right)$ holds.

To determine $\lambda_{m+1}$ for the next iteration step the predicted reduction of the cost function is compared to the actual decrease in $f$. This technique avoids additional timeconsuming solving of the linear system during the trust region radius adaption. The control of $\lambda_{m}$ is described in [13] in detail. For all registrations we start with $\lambda_{0}=16$. The iteration is stopped if the relative reduction of the cost function is less than $2 \%$.

\subsection{Deformable Surface Models}

After initial positioning in a 3D image (see below), a triangular surface mesh is adapted to an image by iteratively carrying out surface detection in the image for each triangle, and reconfiguration of the vertex coordinates by minimizing $E=E_{\text {ext }}+\alpha E_{\text {int }}$. The parameter $\alpha$ weighs the relative influence of an external energy $E_{\text {ext }}$, which drives the mesh towards detected surface points, and an internal energy $E_{\text {int }}$, which maintains the vertex configuration of an initial mesh.

Surface detection is carried out for each triangle center $\mathbf{x}_{i}$. We seek the point $\tilde{\mathbf{x}}_{i}$ along the triangle normal $\mathbf{n}_{i}$ which maximizes the cost function consisting of a feature function $F$ and the distance $j \delta$ to the triangle center according to

$$
\tilde{\mathbf{x}}_{i}=\mathbf{x}_{i}+\delta \mathbf{n}_{i} \underset{j=-l, \ldots, l}{\arg \max }\left\{F\left(\mathbf{x}_{i}+j \delta \mathbf{n}_{i}\right)-D j^{2} \delta^{2}\right\},
$$


where $2 l+1$ is the number of points investigated, $\delta$ specifies the distance between two points on the profile, and $D$ controls the tradeoff between feature strength and distance.

The object specificity of $F$ is crucial to the robustness and accuracy of deformable model adaptation. The image gradient is not a robust lung feature for 4D CT data due to noise, motion artifacts, and many false features inside the lungs. Instead, we search for the point where the grey-value transitions across a particular threshold according to

$$
F(\mathbf{x})= \begin{cases}0 & \text { if } I_{j-1}(\mathbf{x})<I_{\min } \text { and } I_{j}(\mathbf{x})<I_{\min } \\ -1 & \text { if } I_{j-1}(\mathbf{x})>I_{T} \text { and } I_{j}(\mathbf{x})<I_{T} \\ 1 & \text { if } I_{j-1}(\mathbf{x})<I_{T} \text { and } I_{j}(\mathbf{x})>I_{T}\end{cases}
$$

$I_{j-1}(\mathbf{x})$ and $I_{j}(\mathbf{x})$ are two successive grey values on the search profile from inside to outside of the organ, $I_{\min }$ is the minimum grey value of the lung parenchyma, and $I_{T}$ is the transition grey value.

To compute the new vertex coordinates given the detected feature points, we minimize the weighted sum of the external and internal energies. $E_{\text {ext }}$ drives the mesh towards the detected surface points:

$$
E_{\text {ext }}(\mathbf{x})=\sum_{i=1}^{T} w_{i}\left\|\tilde{\mathbf{x}}_{i}-\mathbf{x}_{i}\right\|_{2}^{2}, w_{i}=\max \left\{0, F\left(\tilde{\mathbf{x}}_{i}\right)-D j^{2} \delta^{2}\right\},
$$

$T$ being the number of triangles. The weights $w_{i}$ give the most promising surface points $\tilde{\mathbf{x}}_{i}$ the largest influence during mesh reconfiguration. The internal energy maintains the distribution of the mesh vertex coordinates $\mathbf{v}_{j}$ w.r.t. the edges of a given initial mesh $\tilde{\mathbf{v}}_{j k}=\tilde{\mathbf{v}}_{j}-\tilde{\mathbf{v}}_{k}$

$$
E_{\text {int }}=\sum_{j=1}^{V} \sum_{k \in N(j)}\left\|\mathbf{v}_{j}-\mathbf{v}_{k}-s \mathbf{R} \tilde{\mathbf{v}}_{j k}\right\|_{2}^{2}
$$

where $N(j)$ is the set of neighbors of vertex $j$, and $V$ is the number of vertex coordinates [14]. The rotation $\mathbf{R}$ and the scaling $s$ between original and deforming mesh are estimated in each iteration using a fast closed-form point-based registration method based on singular value decomposition. Since the energies in (6) and 7) are quadratic, energy minimization results in the efficient solution of a sparse linear system using the conjugate gradient method.

\section{Experiments}

\subsection{Image Data and Quantitative Validation Metric}

We assessed the performance of the two algorithms based on a 4D CT study $(512 \times$ $512 \times 165$ voxels, $0.88 \times 0.88 \times 3.0 \mathrm{~mm}$, Philips MX8000 IDT 16-line, retrospectively breathing gated helical cone-beam reconstruction) consisting of $83 \mathrm{D}$ volumes from end inspiration (CT 0) to end expiration (CT 4) to late inspiration (CT 7). Clinical experts carried out 2D slice-based contouring of the lungs and the heart with a commercial 
Table 1. Mesh propagation from 7 CT volumes of different phases (1: early expiration, 7: late inspiration) to end inspiration phase image. Shown are surface distances between the triangulated manual segmentations and the deformed mesh surface before (Initialization) and after volumetric $\mathrm{B}$-spline registration and deformable surface model adaptation respectively. The deformable surface method would require separate modeling of the heart and is not yet available.

\begin{tabular}{|c|c|c|c|c|c|c|c|}
\hline CT No. & 1 & 2 & 3 & 4 & 5 & 6 & 7 \\
\hline \multicolumn{8}{|c|}{ Right Lung (Mean Distance [mm] / 99\%-Quantile Distance [mm]) } \\
\hline Initialization & $1.0 / 6.0$ & $2.0 / 11.8$ & $2.9 / 18.9$ & $3.8 / 29.1$ & $3.4 / 23.3$ & $2.1 / 11.4$ & $1.3 / 9.3$ \\
\hline B-spline & $0.8 / 4.1$ & $1.0 / 5.1$ & $1.2 / 7.0$ & $1.3 / 6.6$ & $1.1 / 5.4$ & $1.5 / 13.1$ & $1.0 / 6.3$ \\
\hline Deformal & $1.2 / 6.4$ & $1.3 / 6.8$ & $1.3 / 7.8$ & $1.3 / 8.2$ & $1.3 / 8.5$ & $1.3 / 8.1$ & $1.2 / 6.5$ \\
\hline \multicolumn{8}{|c|}{ Left Lung } \\
\hline Initializ: & $0.4 / 1.8$ & $1.4 / 5.5$ & $1.9 / 6.3$ & $1.5 / 6.1$ & $1.8 / 6.3$ & $1.8 / 5.7$ & $1.1 / 4.2$ \\
\hline B-spl & $9 / 4.5$ & $1.3 / 8.2$ & $1.5 / 11.6$ & $1.7 / 8.5$ & $1.7 / 9.6$ & $2.1 / 20.5$ & $1.0 / 5.1$ \\
\hline Deformable Models & $1.0 / 4.7$ & $1.1 / 6.1$ & $1.1 / 4.5$ & $1.0 / 4.4$ & $1.1 / 5.1$ & $1.0 / 4.3$ & $1.0 / 4.3$ \\
\hline \multicolumn{8}{|c|}{ Heart } \\
\hline n & $4 / 25.9$ & $2.7 / 10.1$ & $3.2 / 8.4$ & $3.6 / 12.3$ & $3.4 / 13.4$ & $3.3 / 10.9$ & $3.5 / 10.7$ \\
\hline B-spline & $5.2 / 25.7$ & $2.5 / 12.0$ & $3.0 / 12.4$ & $3.1 / 12.3$ & $3.2 / 16.4$ & $2.8 / 9.2$ & $3.7 / 12$ \\
\hline
\end{tabular}

software package. The contours were subsequently transformed to binary masks and their surfaces triangulated. We quantified the difference between the deformed surface meshes and the expert segmentations by calculating the mean and 99\%-quantile distance in $\mathrm{mm}$ between the vertices of the deformed mesh and the expert contours.

\subsection{Experimental Setup}

The CT volume of the end inspiration was used as the template. Patient-specific surface meshes were generated from the expert segmentations for validation. The meshes were deformed using the two methods to match the remaining CT volumes from early expiration to end expiration and compared to the triangulated manually segmented masks.

The B-spline registration was embedded into a multi-scale approach employing both an image pyramid and a parameter pyramid of three levels. The coarsest image resolution level used images of size $75 \times 57 \times 63$ voxels and a grid with $7 \times 7 \times 3$ control points, while the finest level used $256 \times 256 \times 165$ voxels and a $19 \times 19 \times 15$ mesh resulting in 17328 optimization parameters. The computation time was on the order of 50 minutes on average for the registration of two volumes (Intel Pentium IV, 2.66 GHz).

The deformable model was applied on the original image resolution with fixed parameters $l=40, \delta=1 \mathrm{~mm}, D=0.3, I_{\min }=100, I_{T}=600$, and a coarse-to-fine parameter pyramid of $\alpha=1,0.3,0.2$, with 10 iterations on each level. The computation time for the entire adaptation of a high resolution mesh with 5000 vertices (i.e. 15004 parameters) to a volume was on the order of 40 seconds on average (Intel Pentium IV, 2.66 GHz). 


\subsection{Results}

The deformation between end inspiration (CT 0) and the remaining phases was most prominent in the region of the diaphragm and the heart ventricles (Fig. 1 (a,b)). The geometric differences increased to a maximum at end expiration (CT 4) and decreased again towards late inspiration (CT 7) (Table 1, difference between manual segmentations (Initial)).

Both methods captured the overall deformation of the lungs between breathing phases well, with $1.06 \pm 0.04 \mathrm{~mm}(1.26 \pm 0.07 \mathrm{~mm})$ mean difference for the left (right) lung with deformable surface models, $1.43 \pm 0.41 \mathrm{~mm}(1.13 \pm 0.25 \mathrm{~mm})$ mean difference for the left (right) lung with B-spline registration, and $3.35 \pm 0.90 \mathrm{~mm}$ mean difference for the heart with B-spline registration (the surface model approach would require separate modeling of the heart, which has not yet been done). A part of the error can be attributed to inaccuracies in the expert segmentations (inter- and intraobserver variations are typically in the order of $0.1-0.8 \mathrm{~mm}[16]$ ). Small deformations $<1 \mathrm{~mm}$ mean surface distance are beyond the accuracy of both algorithms and cannot be recovered due to noise and the methods' smoothness and shape constraints.

The B-spline method produces partial errors at the diaphragm of the left lung and the heart (Fig. 1 (c,d)). These errors are due to image motion artifacts which cannot be distinguished well from the true lung surface with the SSD similarity measure. The motion artifacts are most severe in the area of the heart because the CT acquisition is breathing-gated, which is not well suited to compensate heart motion. The deformable surface model is more robust w.r.t. artifacts (consistent capturing of deformation for all phases), since the algorithm searches for a certain grey value transition and is thus not affected by motion artifacts. If the image artifacts are less severe (right lung), the Bspline registration outperforms the deformable models because volumetric registration takes more image information into account.

\section{Discussion and Conclusions}

Two alternative methods, a deformable surface method and a B-spline registration algorithm, were established and successfully applied to propagate organs in 4D CT of the chest with good overall mean accuracy of 1.02-1.33 mm and 0.78-2.05 for deformable surfaces and B-splines respectively.

The main advantage of volumetric registration is that the deformation for all organs, including the tumor, can be estimated with a single approach, while organ specific parameter settings and surface models must be designed for every new object addressed with the deformable models. However, the modeling effort pays off by a significant reduction of CPU time (40 seconds vs. 50 minutes) crucial for clinical applicability.

Improvement of the B-spline registration quality may be achieved by considering other similarity measures to better deal with missing image correspondences and image artifacts. Motion artifacts in 4D CT images may be reduced in the future by increased scanner speed and better gating and reconstruction schemes. We also plan to further explore the possibilities of deformable surface models by extension to other organs. An interesting question is the applicability to tumors where shape modeling is difficult. 


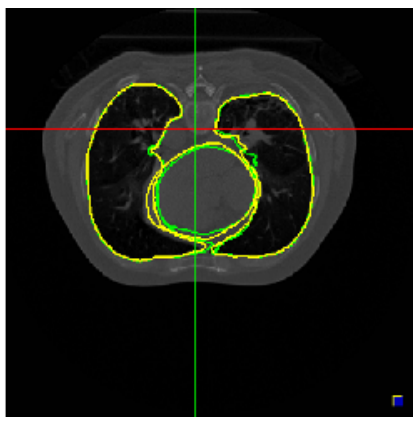

(a) Expert mesh (EE and EI), EE image

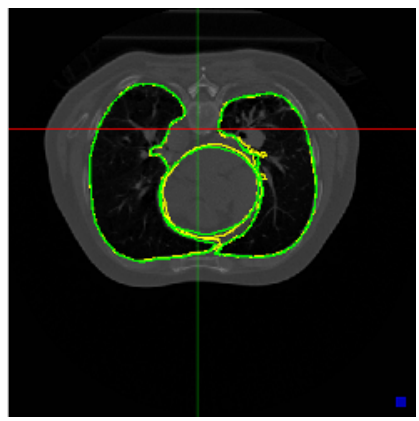

(c) Expert mesh, B-spline warped mesh at EI

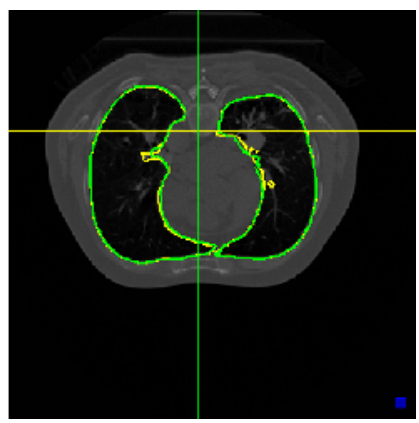

(e) Expert mesh, deformable model at EI

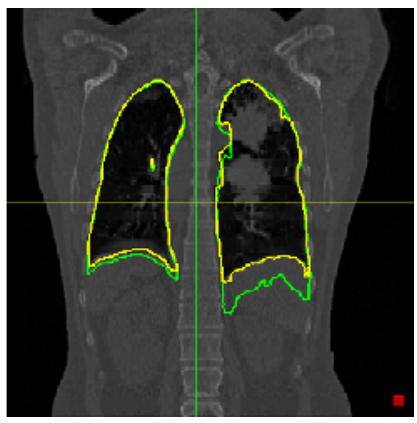

(b) Expert mesh (EE and EI), EE image

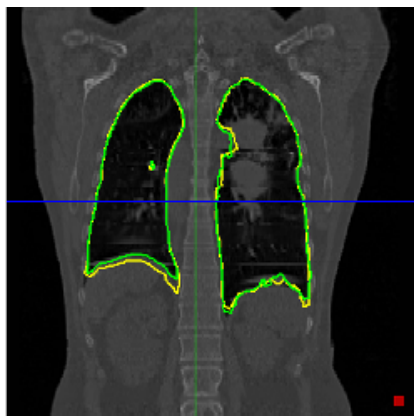

(d) Expert mesh, B-spline warped mesh at EI

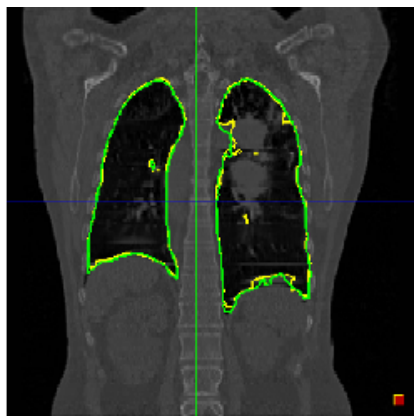

(f) Expert mesh, deformable model at EI

Fig. 1. Expert contours and contours generated by cutting the warped 3D surface meshes with the visible image planes. Significant deformation between end inspiration (EI) and end expiration (EE), (a,b) can be recovered with both the volumetric registration method (c,d) and the deformable surface method (e,f). The heart was only addressed with B-spline registration.

Finally, we need to evaluate more patients for a better understanding of organ and tumor motion and required clinical accuracy. 
Acknowledgements. We thank George Starkshall, PhD, MD Anderson Cancer Center, for the preparation of the CT data of the lung.

\section{References}

1. L. A. G. Ries, M. P. Eisner, and C. L. Kosary et al. (eds). SEER cancer statistics review, 1975-2000. NCI. Bethesda, Maryland, USA [http://www.cancer.gov/statistics].

2. S. S. Vedam, P. J. Keall, V. R. Kini, and R. Mohan. Determining parameters for respirationgated radiotherapy. Medical Physics, 28(10):2139-2146, 2001.

3. L. E. Court and L. Dong. Automatic registration of the prostate for CT-guided RT. Medical Physics, 30(10):2750-2757, 2001.

4. J. V. Hajnal, D. L. Hill, and D. J. Hawkes, editors. Medical Image Registration. CRC Press, Boca Raton, FL, 2001.

5. T. McInerney and D. Terzopoulos. Deformable models in medical image analysis: A survey. Medical Image Analysis, 1(2):91-108, 1996.

6. D. Rueckert, L. I. Sonoda, D. L. Hill, M. O. Leach, and D. J. Hawkes. Nonrigid registration using free-form deformations: Application to breast MRI. IEEE Transactions on Medical Imaging, 18(8):712-721, 1999.

7. E. R. Denton, L. I. Sonoda, D. Rueckert, S. Rankin, C. Hayes, M. O. Leachand, D. L Hill, and D. J. Hawkes. Comparison and evaluation of rigid and non-rigid registration of breast MR images. Journal of Computer Assisted Technology, 23:800-805, 1999.

8. J. Kybic and M. Unser. Fast parametric elastic image registration. IEEE Transactions on Image Processing, 12(11):1427-1442, 2003.

9. D. Perperidis, A. Rao, R. Mohiaddin, and D. Rueckert. Non-rigid spatio-temporal alignment of 4D cardiac MR images. In Second International Workshop on Biomedical Image Registration, Philadelphia, PA, 2003.

10. S. C. Mitchell, B. P. F. Lelieveldt, R. J. van der Geest, H. G. Bosch, J. H. C. Reiber, and M. Sonka. Multistage hybrid active appearance model matching: Segmentation of left and right ventricles in cardiac MR images. IEEE Transactions on Medical Imaging, 20(5):415423, 2001.

11. M. R. Kaus, J. von Berg, W. Niessen, and V. Pekar. Automated segmentation of the left ventricle in cardiac MRI. In International Conference on Medical Image Computing And Computer Assisted Intervention (MICCAI), pages 432-439, 2003.

12. P. Thompson and A. W. Toga. A Surface-Based Technique for Warping Three-Dimensional Images of the Brain. IEEE Transactions on Medical Imaging, 15(4):402-417, 1996.

13. S. Kabus, T. Netsch, B. Fischer, and J. Modersitzki. B-spline registration of 3D images with Levenberg-Marquardt optimization. In Image Processing, SPIE Proceedings, 2004. In press.

14. J. Weese, M. R. Kaus, C. Lorenz, S. Lobregt, R. Truyen, and V. Pekar. Shape constrained deformable models for 3D medical image segmentation. In International Conference on Information Processing in Medical Imaging, pages 380-387, Davis, CA, USA, 2001.

15. P. Thévenaz and M. Unser. Spline pyramids for inter-modal image registration using mutual information. In Image Processing, volume 3169 of SPIE Proceedings, pages 236-247, 1997.

16. D. C. Collier, S. S. C. Burnett, M. Amin, S. Bilton, C. Brooks, A. Ryan, D. Roniger, D. Tran, and G. Starkschall. Assessment of consistency in contouring of normal-tissue anatomic structures. Journal of Applied Clinical Medical Physics, 4(1):17-24, 2003. 